\section{¿Educadores, orientadores, terapeutas? Juventud, sexualidad e intervención social}

\author{
Educators, advisors, therapists? \\ Youth, sexuality, and social intervention
}

Mara Viveros-Vigoya 1

Franklin Gil Hernández ${ }^{1}$

\footnotetext{
${ }^{1}$ Facultad de Ciencias Humanas, Universidad Nacional de Colombia, Bogotá, Colombia.

Correspondencia M. Viveros-Vigoya Departamento de Antropología, Facultad de Ciencias Humanas, Universidad Nacional de Colombia.

Carrera 30 No. 45-03 Edificio 212 Oficina 312 , Bogotá, Colombia. mviverosv@unal.edu.co
}

\begin{abstract}
Sexual and reproductive health programs $d i$ rected at youth have a recent history, related to international politics, especially the International Conference on Population and Development in Cairo, which in 1994 recommended specific services for adolescents and proposed to reduce substantially the pregnancies in this social group. This effort is an opportunity to consider young people as citizens with rights, or also as new clients and health consumers. In this article, we analyze how this conflict is addressed in the Sexual and reproductive health programs, and the limits and range of the mediating role played by civil employees who carry out health interventions, specially when they enter into relationships with other agents of socialization of adolescents (fathers, mothers, educators, etc.), but also with the public health system. In this field we identified the predominant perspectives of social intervention that seeks to direct and structure the possible field of action of youth in sexual matters. These perspectives emphasize individual responsibility in relation to self care and articulate very well with the effective model of health, based on market rationality.
\end{abstract}

Sexuality; Sex Education; Youth

\section{Introducción}

En este artículo analizamos el papel de las y los funcionarios que asumen la intervención social en relación con la salud sexual y reproductiva de los jóvenes en Colombia. Para tal objeto describimos, en primer lugar, la historia de los programas de salud sexual y reproductiva dirigidos a jóvenes y el funcionamiento de dos programas en particular: el Programa de Educación en Sexualidad Humana de la Universidad Nacional de Colombia (UNAL), sede Bogotá, y el Programa de Jóvenes de PROFAMILIA (ver información detallada de los programas más adelante), sede Kennedy. En segundo lugar identificamos el perfil de los funcionarios que asumen las actividades educativas de estos programas, los alcances y límites de su quehacer profesional, asociados con la política pública y el estatuto juvenil. En tercer lugar, analizamos la función de mediación que desempeñan estos actores sociales en relación con otros agentes de socialización (padres y madres, educadores, entre otros). Finalmente, presentamos las perspectivas predominantes en estos proyectos de intervención social, que buscan dirigir y estructurar el posible campo de acción de las y los jóvenes en materia sexual 1 .

La información brindada se basa en la observación y análisis de dos experiencias educativas en sexualidad con jóvenes escolarizados en Bogotá, realizadas por dos entidades, una 
pública y otra privada. En cada una de ellas se efectuaron entrevistas a las y los coordinadores, a los responsables de los talleres y a las personas encargadas de las labores de consejería en sus respectivas instituciones (diez entrevistas en total).

\section{El surgimiento de los programas de salud sexual y reproductiva en Colombia}

Los programas de salud sexual y reproductiva dirigidos a jóvenes en Colombia tienen una historia reciente, bastante relacionada con las políticas internacionales, especialmente con el Programa de Acción de la Conferencia Internacional de Población y Desarrollo de El Cairo, que en 1994 recomendó servicios específicos para adolescentes y propuso reducir sustancialmente todos los embarazos en este grupo social. Esta perspectiva poblacional focalizada forma parte de una tendencia más amplia en el campo de la salud sexual. En el caso colombiano, estos recortes poblacionales corresponden también a las acciones de demanda inducida y protección específica que son de obligatorio cumplimiento (La Resolución 412 de 2000 del Ministerio de Salud de la República de Colombia, establece las actividades, procedimientos e intervenciones de demanda inducida, de obligatorio cumplimiento para EPS - empresas promotoras de salud - y ARS - administradoras de régimen subsidiado - y se adoptan las normas técnicas y guías de atención para el desarrollo de las acciones de protección específica y detección temprana, así como la atención de enfermedades de interés en salud pública). Sin embargo, la elección de este enfoque tiene sus propios motivos: el aumento de la fecundidad y de las ETS/VIH/SIDA en la población adolescente y su baja disposición para usar métodos de protección.

Últimamente se ha hecho un esfuerzo por crear estrategias de salud y educación que les lleguen a los y las jóvenes, especialmente a éstas. Y en casi todos los protocolos de los proyectos de intervención en salud sexual y reproductiva en poblaciones jóvenes las metas prioritarias son reducir la tasa de embarazos adolescentes y la edad de inicio de las relaciones sexuales, como lo muestra también la Política Nacional de Salud Sexual y Reproductiva 2 en su apartado sobre jóvenes.

La llegada de los derechos sexuales y reproductivos trajo nuevas concepciones de las intervenciones en jóvenes, e implicó una adición del tema en los contenidos de los talleres y de la oferta institucional en salud sexual y reproductiva para jóvenes ${ }^{3}$. Pero, $i$ es posible trans- formar la perspectiva inicial de pánico moral ante la sexualidad irresponsable, prevaleciente en las acciones de salud sexual y reproductiva, en una de promoción de la ciudadanía sexual para los y las jóvenes? Por supuesto que sí es posible y deseable este cambio. Sin embargo, los derechos sexuales y reproductivos aún no son la bandera, ni de la política pública, ni de las acciones en el área. Se priorizan las soluciones a las situaciones epidemiológicas cuya importancia no discutimos y se posterga el logro menos tangible de una ciudadanía sexual para los jóvenes y de su empoderamiento en el ejercicio de los derechos sexuales y reproductivos, aunque estas palabras aparezcan frecuentemente citadas en los documentos y protocolos.

Pero también surge una pregunta sobre el carácter que se atribuye a los jóvenes en los programas, en relación con su articulación con un sistema privatizado de la salud. iSon considerados los jóvenes, ciudadanos con derechos, o nuevos clientes, para usar el lenguaje de las últimas reformas en el sistema de salud colombiano promovidas por la Ley 100? Desde la lógica del marketing contemporáneo, los jóvenes son percibidos como unos consumidores con necesidades específicas, como en otras áreas del consumo (ropa, música y otras mercancías). Pero, ¿cuáles son sus posibilidades como ciudadanos, en un mundo que equipara cada vez más el ciudadano con el consumidor 4 ?

Los programas que analizaremos a continuación forman parte de este campo que describimos. Tienen algo en común, la juventud de sus usuarios, y dos diferencias básicas: el uno es de carácter público (aunque con un estatuto especial, por estar suscrito a un programa de salud de una institución universitaria y dirigido a los y las estudiantes) y el otro, privado. El privado ha asumido de manera programática una perspectiva específica para jóvenes, mientras el público atiende a jóvenes considerados como tales, principalmente desde su condición estudiantil.

\section{Programa de Educación en Sexualidad Humana de la División de Salud de la Universidad Nacional de Colombia}

En la década del ochenta fueron creados en la UNAL varios programas, que buscaban pasar de un modelo de tipo asistencial a la promoción de la salud y a la salud comunitaria. Estos programas se organizaron en torno a los componentes de "educación en sexualidad humana”, “concepción y anticoncepción”, “control prenatal” y "prevención y control de ETS/VIH/ SIDA". A partir de una reforma administrativa 
se separó el componente educativo de la atención, y el programa tomó el nombre de Educación en Sexualidad Humana, con el objetivo de "propiciar el análisis de actitudes y valores inherentes al fomento de criterios de autocuidado que contribuyan a la vivencia de una sexualidad sana, responsable y gratificante" 5 (p. 5).

\section{Programa para Jóvenes de Profamilia}

En 1990, la Asociación Pro-Bienestar de la Familia Colombiana (PROFAMILIA) crea su programa Atención Directa a Adolescentes en tres modalidades: los Centros para Jóvenes (en ambientes de atención exclusiva), los Programas para Jóvenes (con espacios reservados a adolescentes dentro de las clínicas para adultos) y los Servicios para Jóvenes (donde comparten espacios y profesionales con la población adulta) 6. Las acciones llevadas a cabo por este programa en la localidad de Kennedy, Bogotá D.C. (el centro de PROFAMILIA en la localidad de Kennedy fue fundado hace 18 años y desde hace 5 años se desarrolla en un programa específico para adolescentes. Kennedy es la localidad de Bogotá D.C. con más población joven. Según cifras de la Alcaldía Municipal, 189.935 jóvenes que representan el 12,5\% del total de la ciudad están en esta localidad. Según datos del Departamento Administrativo Nacional de Estadística de Colombia de 6.422.794 de habitantes de Bogotá D.C. en el 2000, 1.518.718 son jóvenes entre 14 y 26 años, es decir, el 23,6\% de la población total 7) corresponden al proyecto Promoviendo el Acceso de Servicios de Salud Sexual y Reproductiva para Adolescentes auspiciado por la Federación Canadiense de Planificación Familiar. El principal componente del programa son los talleres ofrecidos en instituciones educativas, pero también se efectúan asesorías personalizadas y consultas médicas. Los talleres dados por la orientadora del centro en Kennedy a los colegios de la localidad, si bien tienen una función educativa, se conciben ante todo como el primer paso para acceder a la consulta médica, objetivo substancial de este proyecto.

\section{Las funcionarias}

El título en femenino de este apartado indica que la mayoría de los funcionarios que trabajan en el área de salud sexual y reproductiva son mujeres. Pero este detalle no es anodino, si tenemos en cuenta la lógica compasiva 8, así como ciertas representaciones de la feminidad asociadas al cuidado y a la superioridad moral.
Igualmente, cuando sabemos que estos programas pretenden reeducar, modificar comportamientos y transmitir valores en materia sexual. Agreguemos también que el campo de la salud sexual y reproductiva ha estado históricamente ocupado por mujeres, ya sea sus mentoras o quienes brindan y demandan servicios en este ámbito.

El equipo de siete profesionales del programa universitario está conformado por una médica, una nutricionista, dos enfermeras y tres sicólogas. El equipo de la ONG de salud sexual y reproductiva está integrado también por siete profesionales: una educadora, una trabajadora social, cuatro sicólogas y un sicólogo. Sus trayectorias han sido muy diferentes, aunque se identifican dos tendencias: las de las personas cuya experiencia ha estado siempre en el área de salud sexual y reproductiva y las de quienes provienen de otras experiencias disciplinares y trabajaron en otros campos de la salud hasta llegar a la salud sexual y reproductiva. Las primeras son menos frecuentes que las segundas.

Como vemos, además del sesgo de género, existe un bies disciplinar muy importante. Los equipos están integrados de manera mayoritaria por profesionales de la sicología, saber que es convocado de manera privilegiada para orientar las acciones de salud sexual y reproductiva. Las personas de otras áreas generalmente aportan poco desde sus experiencias disciplinares y asumen tanto los presupuestos teóricos como las técnicas terapéuticas que han aprendido de sus colegas sicólogas. Este sesgo disciplinar también está relacionado con el área de intervención privilegiada por los programas, los individuos, y sólo de forma indirecta, su entorno social.

En general, las personas que trabajan en estos programas demuestran un fuerte compromiso con el tema y una gran vocación, usual en quienes que trabajan en programas de bienestar social. Aunque en este caso la vocación está anclada en el hecho de que la población objetivo de su trabajo es la de los jóvenes. Existe, indudablemente, una búsqueda de un determinado perfil profesional para estos cargos por parte de los programas, particularmente por uno de ellos, como se puede observar en la siguiente afirmación: "Sí hay un perfil. La profesión, la mayoría son sicólogos y hay una trabajadora social. Pero sí debe haber un perfil que sea muy cercano y muy... que les guste trabajar con jóvenes, que tenga muy buena empatía, desde el momento en que dice sigue, pasa. O sea, desde el comienzo, que tenga una actitud de no juzgar a los jóvenes, que comprenda que la se- 
xualidad de los jóvenes es... que la pueden vivir tranquilamente y que nosotros solamente podemos brindar una herramienta para que los jóvenes puedan vivir una sexualidad más placentera y mucho más sana. Ese es el perfil que sí buscamos, que sea muy amigable, que sea muy cercano, que entienda la sexualidad de los jóvenes" (Funcionaria 4).

Se nota que ha habido un esfuerzo, incluso en el área médica, por superar las definiciones de juventud como problema, y por construir visiones más complejas y positivas de esta etapa vital. Sin embargo, persisten representaciones sobre lo juvenil que, en el caso de la salud sexual y reproductiva, continúan asociadas a falencias en la toma de decisiones, en el control de la sexualidad y en la disciplina para usar métodos de anticoncepción. Por eso, entre las características buscadas en los funcionarios encontramos la capacidad de escucha y la disposición para entender a personas que, por lo general, no saben exactamente qué quieren y qué necesitan, asumiendo además que el servicio de consejería ofrecido es el único lugar donde son escuchados y respetados, ya que generalmente viven en un medio hostil a sus particularidades y al ejercicio de su sexualidad. Asî lo indica una de las funcionarias entrevistadas: "Es que el adolescente llega al centro y no sabe, llega asustado, que tuve un retraso, quiere planificar pero no sabe si eso es debido o no lo puede hacer. Además de toda la carga que traen: ¿será que me vieron entrar? Entonces, buscamos que cuando lleguen a los centros tengan un profesional que los escuche, por eso el espacio de asesoría y consejería personalizada hace que se tranquilicen, cuéntame qué te pasa. Y ese servicio de asesoría nos permite hacer una mejor orientación al servicio" (Funcionaria 3).

\section{Alcances y límites del quehacer de las funcionarias}

Las funcionarias enfrentan diversas dificultades en sus actividades profesionales (talleres, asesorías y consultas) que incluyen desde las limitaciones en los recursos institucionales, la precariedad socioeconómica de sus usuarios y el marco del sistema de salud pública, hasta la relación con los diversos actores sociales que participan en el campo de la educación sexual: padres y madres de familia, educadores, líderes religiosos y médicos. Veamos a continuación cómo inciden estas condiciones en su quehacer profesional.

\section{La política pública y el estatuto juvenil}

La juventud se ha convertido en una categoría central en los distintos dispositivos institucionales de intervención social (planes, políticas, consejos, oficinas) que buscan contabilizarla, informarla, asesorarla, subvencionarla, en nuestro caso en relación con los programas de salud sexual y reproductiva. Un reciente estudio sobre la oferta institucional dirigida a la juventud en Colombia indica que en 28 entidades territoriales y en la Nación existe un número importante de ofertas tanto públicas como privadas destinadas explícitamente a los jóvenes a través de proyectos y servicios específicos y, en menor proporción, a través de ofertas públicas 9 .

La juventud se suele definir como una clase de edad delimitada socialmente en torno a una determinada pauta de prácticas sexuales, una relación particular con el trabajo y un tipo de actividades de ocio, entre otras (Martín-Criado E 2005; comunicación personal). Serrano 10 plantea también que la intervención social con los jóvenes ha sido realizada desde una perspectiva que subraya el ajuste o desajuste de sus formas de ser y actuar en relación con las normas del mundo adulto, utilizado como patrón de referencia para calificar lo juvenil. Este "adultocentrismo" habría determinado y legitimado las orientaciones normativas de programas y políticas de salud sexual encaminados hacia los jóvenes. Desde esta aproximación, los deseos y placeres sexuales juveniles han sido concebidos como los de unos sujetos no aptos para responder a los requerimientos de una sexualidad responsable y plena como se supone es la sexualidad adulta. Y los jóvenes, como una población de alto riesgo para la prevención en salud sexual y reproductiva, debido a un inicio sexual desinformado, desprotegido y marcado por la culpa y las desigualdades de género.

A las dificultades asociadas a las percepciones de las y los jóvenes como población definida en términos de carencia, de formación e información, se agrega el hecho de que el adolescente es una figura jurídicamente ambigua, aunque la Constitución de 1991 lo reconoce como sujeto de derechos (el artículo 45 de la Constitución de 1991 establece: "El adolescente tiene derecho a la protección y a la formación integral. El Estado y la sociedad garantizan la participación activa de los jóvenes en los organismos públicos y privados que tengan a cargo la protección, educación y progreso de la juventud"). Las políticas y programas se enfrentan a las diversas ambivalencias del estatuto adolescente y si agregamos la categoría “joven”, el problema jurídico se hace más complejo, ya que 
esta categoría incluye tanto a personas menores de edad como a adultos (la franja etárea que corresponde a la categoría “joven”, abarca el grupo de población entre 14 y 26 años). Por esta razón, las y los funcionarios se ven obligados a acogerse a los marcos normativos jurídico-legales que rigen para los menores de edad en Colombia, de acuerdo a la Convención Internacional de los Derechos del Niño (CIDN) sancionada en la Asamblea General de las Naciones Unidas en noviembre de 1989. La CIDN involucra a la población adolescente en la medida en que cubre a los niños desde el nacimiento y hasta los 18 años de edad (cabe señalar que la CIDN es el primer tratado internacional que reconoce los derechos reproductivos de los adolescentes, cuando establece que es necesario "desarrollar la atención sanitaria preventiva, la orientación a los padres y la educación y servicios en materia de planificación familiar" 11 - p. 32). Así lo ilustra el comentario de una de las funcionarias: “...en la constitución colombiana se habla del niño (...) y se dice que se está trabajando con menores de edad. Estratégicamente es una figura política que permite trabajar con los jóvenes. (...) Entonces un chico de 15 ó 16 está perfectamente cubierto por la política de protección a los niños (...), está con derecho a la educación, con derecho a servicios, a esto y esto. (...) es un niño, pero no lo podemos ver como un niño porque ya está ejerciendo su sexualidad y teniendo relaciones sexuales, pero es éste el marco legal que permite abordar a un adolescente" (Funcionaria 3).

Si bien los menores de 18 años carecen de información sobre los derechos que tienen en el Sistema General de Salud y tienen dificultades de tipo geográfico, cultural y económico para acceder a ellos, la situación se agrava cuando cumplen los 18 años. Este umbral de edad aparece como un punto de quiebre en relación con la cobertura de la seguridad social, ya que a partir de este momento las y los jóvenes pierden sus derechos como beneficiarios del sistema de salud de sus padres, o sus responsables, si éstos la tienen (los jóvenes conservan los beneficios del sistema si demuestran, mediante un certificado, estar estudiando en un establecimiento aprobado por el Instituto Colombiano de Fomento a la Educación Superior). La situación descrita se manifiesta en que "en Colombia, de cada dos jóvenes de 18 años, uno se encuentra excluido del sistema de salud" 12 (p. 20).

Por otra parte, aunque los adolescentes y jóvenes tienen representación como un colectivo social en la construcción de políticas públicas específicas, a través de los consejos de juventud, estos espacios no son suficientes pa- ra la defensa de sus derechos particulares, ni tienen voz ni voto en las políticas y programas que afectan su vida sexual. Si bien algunos grupos pueden auto-considerarse como iguales entre sí, y con problemáticas comunes, debemos tener en cuenta que las otras diferencias de clase, género, etnia o "raza" - no son secundarias a la distinción entre jóvenes y adultos.

La reflexión sobre la ciudadanía y los derechos ya está en el escenario de la salud sexual y reproductiva en Colombia. Pero sigue siendo un componente débil. Si bien en la Política Nacional de Salud Sexual y Reproductiva se habla de fortalecer la participación de las y los jóvenes tanto individual como colectivamente, en la práctica, sabemos que la mayoría de las y los jóvenes tienen muy poca ciudadanía y menos aún en materia sexual y reproductiva. Ante la dificultad de desarrollar estrategias y líneas de acción destinadas a promover la participación de las y los jóvenes como sujetos políticos, la mayor parte de los programas terminan centrándose en el objetivo de promover las responsabilidades individuales de las y los adolescentes en cuanto al autocuidado.

\section{El papel mediador de los funcionarios}

En el compromiso con las políticas y programas de salud sexual y reproductiva para jóvenes incide mucho la generación de espacios de discusión sobre la sexualidad con las y los jóvenes usuarios y con los padres, madres y educadores que están a cargo de su formación, ya que muchos de estos programas no logran generar la recepción social necesaria para garantizar el éxito de estas intervenciones. En la salud sexual y reproductiva salen a relucir temas y enfoques muy diversos relacionados con aspectos morales, religiosos y culturales que se disputan los significados de la "buena moral" en sexualidad 13. Es importante tener en cuenta por ejemplo, la fuerte influencia de la Iglesia Católica en el Estado colombiano, pese a la abolición del Concordato y al reconocimiento constitucional de la pluralidad religiosa. Este influjo ha resultado nefasto en el caso de las políticas de salud sexual y reproductiva, al oponerse a la provisión masiva de preservativos para prevenir la infección del VIH/SIDA y a la disponibilidad de la anticoncepción de emergencia por considerarla una técnica abortiva. Igualmente, al promover la abstinencia (denegando la realidad de la actividad sexual de los jóvenes), estableciendo equivalencias entre servicios preventivos y promoción de inmoralidad y promiscuidad. Estas normas culturales y religiosas conservadoras que se oponen a la 
amplia y universal disponibilidad de información y servicios para jóvenes se constituyen en obstáculos para el ejercicio de una ciudadanía sexual juvenil 13 .

Los programas de salud sexual y reproductiva y sus funcionarios tratan de crear ambientes propicios para que los y las jóvenes puedan ejercer sus derechos sexuales y reproductivos. Sin embargo, se encuentran con una gran limitación: ellos no son los únicos agentes en este campo, otros adultos participan en él con posiciones muy diversas frente a la sexualidad juvenil. Y a veces ellos - los más convencidos del tema - sólo pueden llegar una o dos horas a la semana a una institución educativa, en la que otros adultos, padres y madres de familia, profesores y líderes religiosos, interactúan con estos jóvenes, entorpeciendo la mayoría de las veces los procesos que aquellos funcionarios están tratando de facilitar.

El caso de las acciones de educación sexual es demostrativo de la manera como intervienen estos otros agentes adultos, como lo señalan las siguientes funcionarias: “Es más, la posición de padres y educadores frente a la sexualidad de los adolescentes es literalmente opuesta a lo que manejamos. Para muchos de ellos, el interés del taller es que les digamos a los jóvenes que no tengan relaciones sexuales, que todo sea constreñido, como decía usted. Mi comprensión es totalmente opuesta, es brindar las herramientas para ejercer la sexualidad de manera más tranquila, responsable, enriquecedora" (Funcionaria 6); "Pero el trabajo con padres y con docentes es fundamental y a veces en los colegios hemos visto mucha más resistencia por parte de los docentes que incluso de los mismos padres" (Funcionaria 4).

La situación de dependencia económica de los jóvenes, hace que los adultos no sólo sean quienes solicitan los talleres, sino que - como los pagan - sean quienes decidan qué temas deben incluirse o suprimirse en el taller. Los funcionarios de los programas son a veces convocados a los colegios, por razones que no corresponden a las concepciones sobre sexualidad manejadas por los programas. Una de las funcionarias nos comentó el caso de una solicitud de un taller por parte de las directivas de un colegio femenino preocupadas por el "creciente lesbianismo de las alumnas”. Aunque el programa respondió a la demanda, la coordinadora del taller aprovechó para hablar sobre la sexualidad desde una perspectiva no moralista. Escenarios como éste señalan cuán prioritario es el trabajo con los adultos que interactúan cotidianamente con las y los jóvenes (padres, madres y educadores) para garantizar el respe- to a su voz, necesidades y perspectivas como sujetos de derechos sexuales y reproductivos y como activos partícipes en su ejercicio, promoción y defensa.

La situación de dependencia económica de las y los jóvenes es muy limitante también para el ejercicio del derecho a la confidencialidad. Los, y particularmente las jóvenes, van casi siempre de manera clandestina, a solicitar una asesoría en los programas, a obtener un método de planificación, a buscar una anticoncepción de emergencia o a hacerse una prueba de embarazo. En algunas ocasiones, ciertos padres de familia, enterados de que sus hijos o hijas han asistido a estos programas no sólo exigen ver sus historiales clínicos, sino que acusan a los funcionarios de complicidad con lo que perciben como "libertinaje sexual de los y las jóvenes”. Al respecto, una funcionaria nos relata: "Por derecho, la historia clínica se le entrega a la persona, (...) de resto nadie, nadie, tiene por qué ver ese texto. El médico, pero de resto nadie. Y eso se lo hemos dicho a los padres cuando vienen aquí. Y arman todo el alboroto y que somos alcahuetas, que estamos incitando a los hijos a tener relaciones sexuales, que con razón ellos se volvían promiscuos. Pero así hagan una pataleta nosotros no le damos información no autorizada por [el joven]" (Funcionaria 4).

En resumen, la falta de implicación de padres y educadores en estos programas explica algunos de sus límites, ya que los cambios promovidos en los jóvenes son individuales y fundamentalmente actitudinales. No se generan entornos sociales, escolares y familiares que contribuyan al fortalecimiento y sostenibilidad de esos estilos de vida saludables propuestos. En esta perspectiva se está intentando incrementar el número de las acciones educativas para padres y educadores. Por el momento se está experimentando, en algunas localidades, el modelo de pares multiplicadores con padres y madres de familia. La analogía del sándwich que hiciera una de las funcionarias para describir la situación en la que se encuentran muchas y muchos jóvenes nos permite identificar las tensiones en medio de las cuales se da su inicio sexual y anticonceptivo, y la ambivalencia de los contenidos formativos que reciben. Según describe ella: "Los jóvenes están como en medio de un sándwich en que por un lado están sometidos a la presión del grupo y de los medios de comunicación que les dicen 'debes tener ya tus relaciones sexuales y si no las tienes eres un bobo' y por el otro lado tienen a la familia y a la escuela que les dicen 'usted no tiene por qué saber de esto"' (Funcionaria 4). 


\section{Reflexiones finales}

Las funcionarias que asumen las tareas esenciales de estos proyectos educativos, independientemente de su formación académica inicial, buscan reforzar las destrezas de los y las jóvenes para auto-controlarse, adaptándose a las definiciones normativas de la sexualidad 1,14 . Sin embargo, no cuestionan el orden de género y de generación que les impide el ejercicio pleno de su ciudadanía sexual. De igual manera, pese a su mejor voluntad, continúan reproduciendo las orientaciones del modelo anterior de control natal, cuyo interés fundamental es disminuir la fecundidad juvenil. Lo nuevo es que ellas lo hacen en un contexto que valora el aplazamiento del inicio sexual y empieza a introducir la abstinencia sexual como mecanismo necesario para contrarrestar el aumento de la fecundidad juvenil, en consonancia con las directrices actuales de las influyentes políticas norteamericanas en el campo de la salud sexual y reproductiva. (Este discurso a favor de la abstinencia ha tomado tanta fuerza que incluso logró filtrarse en la formulación de la Política Nacional de Salud Sexual y Reproductiva y afectar la financiación de algunas actividades de anticoncepción propuestas por PROFAMILIA).

Las perspectivas predominantes en estos proyectos educativos han sido psicológicas y médicas, pese al reconocimiento de distintos aspectos involucrados en la sexualidad (en Colombia se creó el Plan Nacional de Educación Sexual, liderado por el Ministerio de Educación desde el año de 1993. Su evaluación, realizada en 1998, señala la persistencia de temores y resistencia de parte de maestros y padres en relación con su abordaje de forma directa y explícita y "la consecuente capacitación deficiente de quienes tienen la responsabilidad de su implementación" 15 (p. 27). Igualmente, destaca el énfasis de la información en los riesgos del ejercicio sexual, principalmente los relacionados con los embarazos precoces y las enfermedades de transmisión sexual). Los enfoques sicológicos se han expresado en la percepción de la actividad sexual como una manifestación de la vida psíquica regida por leyes propias 16, y en la orientación de los contenidos educativos hacia el fortalecimiento de habilidades psico-sociales para la elección de métodos de anticoncepción y protección, y de actitudes activas hacia el autocuidado. Los enfoques médicos se han manifestado en la redefinición de las prácticas sexuales de las y los jóvenes en términos patológicos, convirtiéndolos en comportamientos de riesgo, susceptibles de exigir exámenes, diagnósticos y tratamientos y motivar políticas de salud pública.

Estas dos perspectivas proponen una aproximación individualizante y normativa a la sexualidad juvenil que busca generar en cada joven la capacidad de evaluar sus comportamientos sexuales con razonamientos prescriptivos, así sean los de ejercer una sexualidad "libre y satisfactoria”, pero "responsable y sana”. No se puede ignorar que este énfasis en las responsabilidades individuales en relación con el autocuidado se articula muy bien con el modelo vigente de salud, fundado en la racionalidad del mercado, sus esquemas de análisis y sus criterios de decisión 1. La privatización de la salud trae aparejada una ideología de la competencia que justifica las desigualdades sociales en el acceso a los servicios de salud y los distintos grados de morbi-mortalidad relacionados con la sexualidad y la reproducción. Por eso, aunque en el papel se señale el nexo existente entre estas desigualdades y la pobreza, el lugar de residencia y actualmente, la situación de desplazamiento, en la práctica, los programas y servicios parecen ignorar estas circunstancias, y aceptar de forma implícita su carácter ineluctable. Tal vez, porque su margen de acción es muy reducido y el nivel de frustración generado por las nuevas situaciones sociales es muy alto 17 . Sin embargo, mientras no se integre una perspectiva clara de derechos a los programas de salud sexual y reproductiva, la posibilidad de transformar esta situación desaparecerá doblemente, como posibilidad objetiva, concretada en un conjunto de disposiciones, mecanismos y reglamentos que permitan garantizar los derechos sexuales y reproductivos de los jóvenes, y como posibilidad subjetiva, como derechos de los cuales pueden realmente apropiarse. 


\section{Resumen}

Los programas de salud sexual y reproductiva dirigidos a jóvenes tienen una historia reciente, relacionada con las políticas internacionales, especialmente la CIPD de El Cairo, que en 1994 recomendó servicios específicos para adolescentes y propuso reducir sustancialmente los embarazos en este grupo social. Esta focalización es una oportunidad para considerar a los jóvenes como ciudadanos con derechos, o también como nuevos clientes con consumos específicos en salud. En este artículo analizamos cómo se expresa este conflicto en los programas de salud sexual y reproductiva, y los límites y alcances del papel mediador que desempeñan los funcionarios que realizan intervenciones en salud, especialmente cuando entran en relación con otros agentes de socialización (padres y madres, educadores, etc.) de los y las adolescentes, pero también con el sistema público de salud. En este campo identificamos las perspectivas predominantes de intervención social que buscan dirigir y estructurar el posible campo de acción de las y los jóvenes en materia sexual. Estas perspectivas hacen énfasis en las responsabilidades individuales en relación con el autocuidado y se articulan muy bien con el modelo vigente de salud, fundado en la racionalidad del mercado.

Sexualidad; Educación Sexual; Juventud

\section{Referencias}

1. Viveros M. El gobierno de la sexualidad juvenil y la gestión de las diferencias. Reflexiones a partir de un estudio de caso colombiano. Revista Colombiana de Antropología e Historia; en prensa.

2. Ministerio de la Protección Social, República de Colombia. Política Nacional de Salud Sexual y Reproductiva. Bogotá DC: Ministerio de la Protección Social; 2003.

3. Gil F. Proyecto de investigación "Evaluación cualitativa de programas de SSR en poblaciones jóvenes de Bogotá y Cali (una mirada de género, clase, 'color de piel' y orientación sexual)". Bogotá: Centro de Estudios Sociales, Escuela de Estudios de Género, Universidad Nacional de Colombia; 2004.

4. García-Canclini N. Consumidores y ciudadanos: conflictos multiculturales de la globalización. México DF: Grijalbo; 1995.

5. Cifuentes N. Programa de salud integral. Hacia una cultura de la salud. Informe. Bogotá: División de Salud Estudiantil, Dirección de Bienestar Universitario, Universidad Nacional de Colombia; 2003.

6. Asociación Pro-Bienestar de la Familia Colombiana. Centro para jóvenes. Presentación de una experiencia. Bogotá: Fundación Restrepo Barco; 2000.

7. Departamento Administrativo de Acción Comunal y Econometría S.A. Línea de base sobre la situación actual de la juventud del Distrito. Bogotá: Alcaldía Mayor; 2001.

8. Fassin D. Des maux indicibles. Sociologie des lieux d'écoute. Paris: La Découverte; 2004.

9. Asociación Scouts de Colombia. Análisis de oferta institucional en ejecución para la adolescencia y la juventud en Colombia. Bogotá: Editorial SIJU; 2004.

\section{Colaboradores}

La concepción y diseño, la escritura del artículo, la revisión de los borradores y la responsabilidad académica final del artículo son de M. Viveros-Vigoya. Sin embargo, F. G. Hernández aportó insumos sustanciales al artículo, recogió y analizó datos sustantivos para el mismo y participó desde el inicio en la discusión sobre el diseño e interpretación de los datos presentados.

10. Serrano JF, Bejarano L, Caicedo A, Hoyos D, Quintero F. Estado del arte de la investigación sobre juventud para la formulación de la política. Informe de investigación. Bogotá: Departamento Administrativo de Acción Comunal, Departamento de Investigaciones de la Universidad Central; 2002.

11. Checa S. Introducción. Aproximaciones a la problemática de la sexualidad adolescente. In: Checa S, editor. Género, sexualidad y derechos reproductivos en la adolescencia. Buenos Aires: Paidós Tramas Sociales; 2003. p. 19-35.

12. Presidencia de la República de Colombia. Política Nacional de Juventud: bases para el plan decenal de juventud 2005-2015. Bogotá DC: Presidencia de la República de Colombia; 2004.

13. Shepard B. Pecado y derechos humanos. La falta de ciudadanía sexual de la juventud. In: Cáceres C, Frasca T, Pecheny M, Terto V, editores. Ciudadanía sexual en América Latina. Abriendo el debate. Lima: Universidad Peruana Cayetano Heredia; 2004. p. 141-53.

14. Foucault M. Tecnologías del yo y otros textos afines. Barcelona: Paidós; 1991.

15. Secretaría Distrital de Salud de Bogotá. Modelo de servicios de atención integral en SSR para adolescentes y jóvenes del Distrito Capital. Bogotá: Secretaría Distrital de Salud de Bogotá; 2004.

16. Bozon M. Sociologie de la sexualité. Paris: Nathan; 2002.

17. Bourdieu P. La misère du monde. Paris: Editions du Seuil; 1993.

Recibido el 01/Jul/2005

Aprobado el 12/Ago/2005 